\title{
Analytischer Rahmen zur Erfassung von Themenkarrieren in der Wissenschaft
}

In diesem Kapitel wird aufbauend auf den vorangegangenen Kapiteln zu Transformationen in der Wissenschaft (Kp. 2), zur Entstehung von Themen (Kp. 3) und zur Funktionsweise der Wissenschaft (Kp. 4) die theoretische Perspektive entwickelt, die zur Analyse von Themenkarrieren in der Planungswissenschaft eingenommen wird. Es erfolgt also die Selektion, Übersetzung und Anpassung der dargelegten Erkenntnisse aus dem Theorieteil mit dem Ziel, die eigene empirische Analyse zu leiten und die Beantwortung der Frage zu ermöglichen.

Es geht dabei nicht um die Darlegung eines in sich geschlossenen Erklärungsmodells. Vielmehr wird eine Forschungsheuristik bzw. ein analytischer Bezugsrahmen expliziert, die/der als Interpretationsfolie die geschilderten Theorien und Modelle für die Analyse der empirischen Befunde strukturiert und nutzbar macht. Im Sinne eines "sozialwissenschaftlichen Kategoriengerüsts“ (Benz 2011: 99) stellt diese Forschungsheuristik ein (relativ allgemeines) Raster von Konzepten bereit, das der Erfassung und Einordnung empirischer Beobachtungen dient und das es ermöglicht, jene Erklärungsgegenstände zu untersuchen, die in den vorangegangenen Kapiteln (2-4) als potenziell relevant für die Beantwortung der Forschungsfrage identifiziert wurden (Mayntz und Scharpf 1995: 39). Die Heuristik fungiert als Scheinwerfer, indem sie die Aufmerksamkeit auf bestimmte Aspekte lenkt, die wahrscheinlich ein hohes Erklärungspotenzial besitzen, und andere Aspekte zurückstellt, die weniger vielversprechend erscheinen (Scharpf 2006: 64). Ein solches Selektionsprinzip liegt in unterschiedlicher Ausprägung jeder (wissenschaftlichen) Beobachtung unweigerlich zugrunde (Popper 1993: 354-375), gleichwohl bestimmt es bereits maßgeblich über den Prozess und das Ergebnis der Forschung. Ziel dieses Kapitels ist es daher, die theoretischen Vorannahmen und analytischen Prämissen weitestgehend offenzulegen und zu begründen. Die Definition zentraler Begriffe und Konzepte soll ein gemeinsames

A. Gravert, Themenkarrieren in der Wissenschaft, 
Verständnis zwischen Autor und Leser*in erzeugen, Klarheit in der Argumentation gewährleisten und eine Verbindung der vorliegenden Forschungsarbeit mit schon vorhandenen Erkenntnissen herstellen.

Die Explikation der Forschungsheuristik verläuft in fünf Abschnitten vom Allgemeinen zum Konkreten. Zunächst werden zentrale Konzepte des zu rekonstruierenden Makrophänomens - Themenkarrieren in der Wissenschaft - definiert und erläutert (Kp. 5.1). Für die Rekonstruktion wird auf das Kategoriengerüst des Akteurzentrierten Institutionalismus sowie dessen Prinzip der Mikrofundierung von Makrophänomenen zurückgegriffen. Dieses Gerüst, einschließlich des dieser Arbeit zugrunde liegenden Verständnisses von Struktur und Akteur*in sowie von der wechselseitigen Dynamik zwischen diesen beiden Polen, wird anschlieBend dargelegt (Kp. 5.2). Im dritten Schritt wird der institutionelle Kontext des Systems Wissenschaft und dessen Einbettung in die gesellschaftliche Umwelt skizziert (Kp. 5.3). Im Anschluss wird auf wissenschaftliche Akteur*innen und deren Handlungskontext eingegangen (Kp. 5.4). Zuletzt werden die Erkenntnisse in Bezug auf die Themenwahl wissenschaftlicher Akteur*innen konkretisiert und systematisiert (Kp. 5.5).

\subsection{Themen, Aufmerksamkeit und Themenkarrieren in der Wissenschaft}

Die Begriffe ,Thema', ,Aufmerksamkeit' und ,Themenkarriere' sind im Rahmen dieser Arbeit eng miteinander verknüpft und lassen sich nicht ohne Bezug aufeinander konzeptualisieren. Eine einheitliche Auslegung dieser Begriffe im öffentlichen wie im wissenschaftlichen Diskurs wird in der Regel implizit vorausgesetzt und auf eine Definition verzichtet. Im Sinne der analytischen Präzision und der empirischen Operationalisierung sowie zur Schaffung eines gemeinsamen Verständnisses des Forschungsgegenstandes ist eine Begriffsbestimmung an dieser Stelle jedoch unverzichtbar. Benötigt werden Konzepte, mit denen die sozialen Mechanismen der Themenentwicklung in der Planungswissenschaft über einen Zeitraum von mindestens zwei Jahrzehnten nachvollzogen werden können. Die Erläuterung und Abgrenzung der Begriffe baut auf den in den vorangegangenen Kapiteln geschilderten Erkenntnissen auf und übersetzt die primär aus den Medien- und Kommunikationswissenschaften stammenden Konzepte aus Abschnitt 3.1 und 3.2 in das theoretische Bezugssystem der Wissenschaftssoziologie (siehe insb. Kp. 4).

Grundsätzlich ist für die Übertragung der in Kapitel 3 erläuterten Thematisierungsmodelle und -begriffe auf die Wissenschaft ein zentraler Unterschied im 
Hinblick auf den jeweiligen Kontext zu berücksichtigen. Die Ansätze aus Kapitel 3 bewegen sich ausnahmslos in dem Dreieck Medien, Öffentlichkeit, Politik (Rössler 2015). Diese Systeme unterliegen bestimmten Funktionsweisen und sie haben untereinander bestimmte Formen der strukturellen Kopplung, die sich von jenen der Wissenschaft (mit anderen Systemen) unterscheiden. Die Wissenschaft verfügt zudem über das einmalige Charakteristikum, dass jede*r wissenschaftliche Akteur*in Rezipient*in und Verfasser*in, Beeinflusser*in und Beeinflusste*r zugleich ist. Wissenschaftsverlage etwa vermitteln im Gegensatz $\mathrm{zu}$ anderen Kultur-vermittelnden Wirtschaftsorganisationen, wie bspw. Kunstgalerien, Plattenfirmen, Radiosendern oder Zeitungsverlagen nicht zwischen Kulturschaffenden und einem Laienpublikum. Stattdessen haben sie es auf der Zulieferer- wie auf der Abnehmerseite mit Wissenschaftler*innen, also mit Kollaborateur*innen und Konkurrent*innen im Wettstreit um wissenschaftliche Autorität zu tun (Schimank und Volkmann 2012: 166). Diese Systemunterschiede gilt es nicht nur bei der Definition elementarer Begriffe in diesem Kapitel, sondern auch bei der Übertragung weiterer Konzepte auf die Wissenschaft in den Ergebniskapiteln 9 und 10 zu beachten.

Die Konzeptualisierung von ,Thema' in der Kommunikationssoziologie ist auch auf die Wissenschaft anwendbar. Dementsprechend ist ein wissenschaftliches Thema ein Sinnkomplex, der als Struktur jeder wissenschaftlichen Kommunikation zugrunde liegt und der bei den Kommunikationsteilnehmenden ein gemeinsames Wissen erzeugt. Themen überdauern Einzelkommunikationen bzw. Beiträge und fassen diese zu übergeordneten, entwicklungsfähigen Sinnkomplexen zusammen. Der Sinnzusammenhang wird durch die Kommunikation darüber hergestellt, reproduziert und verändert. Als Erwartungsstruktur vermittelt das Thema den Kommunikationsteilnehmenden einen Bereich dessen, was gesagt werden kann und was nicht bzw. was zum Thema passt und was nicht. Nichtsdestotrotz gibt es (zu einem bestimmten Zeitpunkt) so viele Verständnisse eines Themas, wie es Teilnehmende der Kommunikation gibt. Als wissenschaftliche Beiträge sind wissenschaftliche Publikationen, Vorträge, Forschungsanträge, aber auch Kommunikationen im Rahmen informeller Gespräche zu fassen. Ein Forschungsgegenstand wird zum Thema, sobald er zum Objekt fachöffentlicher Kommunikation wird.

Ein wissenschaftliches Thema ist durch dessen zeitliche, inhaltliche, soziale, politische und räumliche Dimension strukturiert. Es nimmt einen zeitlichen Verlauf, indem es mit jeder neuen Kommunikation aktualisiert wird und somit die ihm zugeschriebene Bedeutung ändert und die mit ihm verbundenen Akteur*innen und Interessen auf unterschiedliche Weise aufruft. Es ist als inhaltliches Bezugssystem oder Bedeutungsnetzwerk zu verstehen, indem es 
Themen, Ereignisse, Objekte oder Akteur*innen in einen (themenspezifischen) Sinnzusammenhang ordnet. Auf der inhaltlichen Ebene lässt sich ein Thema als quasi-hierarchisches Netzwerk fassen, das verschiedene Sub-Themen bzw. untergeordnete Themen in einen Zusammenhang bringt, genauso wie es selbst Teil mindestens eines weiteren, übergeordneten Themas ist. Ein Thema ist sozial strukturiert, indem es Akteur*innen aktiviert und zusammenbringt, aber auch Akteur*innen von der Kommunikation ausgrenzt, die keinen zum Thema passenden Beitrag leisten (können). Die Aufmerksamkeit für und Deutungshoheit über ein Thema ist umkämpft und an die Verfügbarkeit von Ressourcen geknüpft, weshalb es auch eine politische Dimension besitzt. Die Verhandlung von Themen ist mit Interessen verbunden, indem Akteur*innen ein Thema strategisch aufgreifen, hervorheben, in ihrem Sinne rahmen oder meiden können. Die zeitliche, inhaltliche, soziale und politische Dimension ist zudem - zumindest in der Planungswissenschaft - an die räumliche Verortung geknüpft. Das heißt, in welcher Form, wann und durch wen ein Thema in der Wissenschaft aufkommt, hängt auch von lokal spezifischen Gelegenheitsstrukturen ab, die sich aus der Topologie, ortsgebundenen Netzwerken und den Regelungssystemen ableiten.

,Fachöffentliche Aufmerksamkeit" wird verstanden als das Aufwenden knapper Ressourcen - Zeit, ökonomisches Kapital, Sozialkapital und weitere - seitens der Fachgemeinschaft darauf, bestimmte Informationen zu prozessieren und darüber im wissenschaftlichen Kontext zu kommunizieren. In Abgrenzung zur individuellen, kognitiven Aufmerksamkeit wird fachöffentliche Aufmerksamkeit in dieser Arbeit als ein soziales, zwischenmenschliches Phänomen betrachtet, das erst in der Kommunikation über das Objekt der Aufmerksamkeit emergiert. Über einen Zeitraum betrachtet, steht fachöffentliche Aufmerksamkeit gegenüber einem Thema daher für die Intensität, mit der darüber seitens der Fachgemeinschaft(en) kommuniziert wird (Klamer und van Dalen 2002: 296). Diese Intensität lässt sich anhand der Frequenz thematisch einschlägiger, formeller Kommunikationen - bspw. Publikationen, Zitationen, Konferenzen, Vorträge - sowie der Anzahl der Kommunikationsteilnehmenden näherungsweise bestimmen. Dementsprechend lässt sich anhand dieser Merkmale eine Aufmerksamkeitskurve über einen Zeitverlauf abtragen.

Das Erregen fachöffentlicher Aufmerksamkeit ist ein Erfordernis zur Erlangung von Reputation. Dabei ist die insgesamt verfügbare fachgemeinschaftliche Aufmerksamkeit - im Gegensatz zur individuellen, kognitiven Aufmerksamkeit, die großen Schwankungen unterliegt und ermüden kann - weitestgehend konstant und ändert sich nur insoweit, wie sich auch die Größe der Fachgemeinschaft und der Umfang ihrer Kommunikation ändern. Weil eine große Zahl wissenschaftlicher Akteur*innen und ein unerschöpflicher Fundus an Themen um 
ein gleichbleibendes $\mathrm{Ma} ß$ an wissenschaftlicher Aufmerksamkeit konkurriert, ist Aufmerksamkeit ein knappes und begehrtes Gut.

Eine ,Themenkarriere ' in der Wissenschaft umfasst den zeitlichen Verlauf der Aufmerksamkeit seitens einer oder mehrerer wissenschaftlicher Fachgemeinschaft(en) gegenüber einem bestimmten Thema, das über einen gewissen Zeitraum hinweg im fachöffentlichen Diskurs etabliert war, das also zwischenzeitlich ein großes Maß an Aufmerksamkeit erreicht hat. Der Begriff Themenkarriere bezieht sich demzufolge auf die gesamte Entwicklung eines Themas, setzt aber eine Phase der Etablierung voraus. Demgegenüber kommen die Begriffe ,Themenverlauf' oder ,Themenentwicklung ' ohne die zwischenzeitliche Etablierung aus. Unter ,Thematisierung ' wird das Aufgreifen des Themas in den wissenschaftlichen Beiträgen der Mitglieder einer Fachgemeinschaft verstanden.

Wissenschaftliche Themenkarrieren durchlaufen unterschiedliche Phasen, in denen sich nicht nur ihre Wirkmächtigkeit, sondern auch inhaltlich-argumentative Deutungen, eingebundene Akteur*innen und institutionelle Rahmenbedingungen wandeln. Themenkarrieren in der Wissenschaft können - wie solche in den Medien oder in der Politik - einem zyklischen Verlauf folgen. Gravert, Günzel und Wiechmann (2019) identifizieren drei aufeinanderfolgende Phasen: eine Latenzphase mit geringer Aufmerksamkeit, eine fünf bis siebenjährige Fokusphase mit hoher Aufmerksamkeit und eine Normalisierungsphase, in der die Aufmerksamkeit - auf ein gegenüber der Latenzphase deutlich erhöhtes Niveau absinkt. Zusätzlich wird im Rahmen dieser Arbeit eine ein- bis zweijährige Durchbruchphase vor der Fokusphase abgegrenzt, in der die Aufmerksamkeit von dem niedrigen und vergleichsweise stabilen Niveau der Latenzphase auf das beständig hohe Niveau der Fokusphase gehoben wird. Die separate Betrachtung dieser Durchbruchphase betont die Bedeutung dieses Segments und ermöglicht eine ausführlichere Analyse entscheidender Prozesse der Themenentstehung sowie des damit verbundenen institutionellen Wandels.

Eine wissenschaftliche Themenkarriere ist - analog zum Themenbegriff - zeitlich (s. o.), inhaltlich, sozial, politisch und räumlich strukturiert. Eine inhaltliche Verschiebung, Verkürzung oder Erweiterung eines Themas kann sich auf die fachöffentliche Thematisierung auswirken, indem sie bspw. eine Erweiterung des Akteur*innenkreises, der einen Beitrag zu dem Thema leisten kann, ermöglicht. Umgekehrt verändern Akteur*innen und ihre Beiträge ein Thema, indem etwa neu aufgerufene Akteur*innenkreise im Verlauf der Themenkarriere auch eine Bedeutungsverschiebung erwarten lassen. Sozial und politisch determiniert ist eine Themenkarriere, weil die Akteur*innen das Thema vor dem Hintergrund ihrer individuellen Handlungsziele und Umsetzungschancen aufgreifen, vorantreiben, verändern oder vermeiden. Umgekehrt wirkt sich eine Themenkarriere auf die 
Sozialstruktur der Fachgemeinschaft und die Handlungsoptionen ihrer Mitglieder aus, indem etwa ein plötzlich relevantes Thema die Allokation von Ressourcen modifiziert, Kooperationsnetzwerke umformt und neue Karrierestationen eröffnet. In der Planungswissenschaft hat eine Themenkarriere eine räumliche Komponente, denn indem die Disziplin sich mit der empirischen und theoretischen Erforschung räumlicher Entwicklung und Planung befasst, ist die Entfaltung eines Themas im Rahmen der Wissensproduktion untrennbar mit der räumlichen Ausdehnung und der lokal spezifischen Interpretation des untersuchten Phänomens verbunden.

Eine Themenkarriere in der Wissenschaft entsteht also, wenn eine signifikante Anzahl von Mitgliedern einer Fachgemeinschaft ein bestimmtes Thema aufgreift und in kurzen Intervallen wissenschaftlich darüber kommuniziert. Diese Häufung von Kommunikationen lässt sich empirisch - quantitativ und qualitativ nachvollziehen. Für die kausale Rekonstruktion von Themenkarrieren ergibt sich darüber hinaus die Frage, welche Mechanismen dazu führen, dass wissenschaftliche Akteur*innen ein bestimmtes Thema aufgreifen und darüber kommunizieren (und über ein anderes nicht).

\subsection{Struktur und Akteur*innen}

In dem dieser Arbeit zugrundeliegenden Akteur*inverständnis werden Entscheidungen, die den Forschungsinhalt, mithin die Themenwahl, unmittelbar bestimmen, (1.) immer durch das wissenschaftliche Individuum selbst (2.) vor dem Hintergrund eines institutionellen Kontextes getroffen. Die Entscheidung zugunsten oder zulasten von Problemen, Methoden und Vorgehen sind in variierendem Grad durch die institutionellen Rahmenbedingungen beeinflusst, es ist aber dennoch das Individuum, das die Entscheidung trifft. Jegliche Einflussnahme auf den Forschungsinhalt muss also durch die Entscheidungen wissenschaftlicher Individuen vermittelt sein. In der Sprache der Akteur-Netzwerk-Theorie wird der*die Forschende insofern zum „obligatory point of passage“ (Latour 1993: 43-44; Gläser 2012a: 5).

Von diesem Verständnis ausgehend wird zur Beantwortung der Forschungsfrage und zur Einordnung in den Theoriekontext im Folgenden die Perspektive des Akteurzentrierten Institutionalismus eingenommen (Mayntz und Scharpf 1995). Dieser stellt einen generellen theoretischen Bezugsrahmen einschließlich zentraler Konzepte bereit und bietet ein grundlegendes Verständnis für Akteur*in-Struktur-Dynamiken, das zur Erklärung des Handelns von Wissenschaftler*innen herangezogen werden kann. Der Ansatz stellt eine erprobte und in 
der planungswissenschaftlichen Disziplin bekannte Nomenklatur zur Verfügung, die ein gemeinsames Verständnis zwischen Autor und Leser*in erzeugen und die fallübergreifende Vergleichbarkeit von Annahmen und Forschungsergebnissen gewährleisten soll.

Der Ansatz ist offen gegenüber differenzierungstheoretischen Perspektiven, sodass Wechselwirkungen zwischen der Planungswissenschaft und anderen gesellschaftlichen Teilsystemen, etwa der Politik oder den Massenmedien, analytisch erfasst werden können (Mayntz et al. 1995: 48). Darüber hinaus bietet der Akteurzentrierte Institutionalismus eine Verknüpfung zwischen institutionalistischen und netzwerktheoretischen Ansätzen, die für die Rekonstruktion von Themenkarrieren im Rahmen dieser Arbeit genutzt wird. So werden Netzwerkbeziehungen als Sozialkapital erfasst, das Akteur*innen bestimmte Handlungen ermöglicht (Scharpf 2006: 233-240). Netzwerke sind in diesem Sinne Gelegenheitsstrukturen sowie gleichzeitig Machtstrukturen, wenn bspw. „ein Mitglied einer dyadischen Austauschbeziehung Zugang zu alternativen Ressourcenquellen hat, das andere Mitglied jedoch nicht" (Scharpf 2006: 237). In Bezug auf eine*n einzelne*n Akteur*in lässt der Akteurzentrierte Institutionalismus mehrere (teilweise konkurrierende) Systembezüge zu, was eine insbesondere für die Untersuchung der Planungswissenschaft wichtige analytische Option darstellt. So kann ein Individuum bspw. aus der Perspektive eines Mitglieds einer wissenschaftlichen Fachgemeinschaft handeln, aber auch in seiner*ihrer Funktion als Leiter*in eines privatwirtschaftlichen Planungsbüros, sofern es die Situation erlaubt (vgl. Mayntz et al. 1995: 52).

Der Akteurzentrierte Institutionalismus ordnet sich in die Renaissance institutionalistischer Ansätze seit den 1960er Jahren ein und knüpft dabei primär an den politikwissenschaftlichen Neo-Institutionalismus an (Mayntz et al. 1995: 40-43). Er zeichnet sich dadurch aus, dass er den strategischen Handlungen und Interaktionen zweckgerichteter Akteur*innen die gleiche Bedeutung beimisst wie den ermöglichenden und beschränkenden Effekten institutioneller Strukturen (Scharpf 2006: 72). So führt der Ansatz institutionenzentrierte und akteur*innenzentrierte Herangehensweisen, institutionalistische und handlungstheoretische Paradigmen zusammen und gewinnt dadurch „eine größere Übereinstimmung zwischen den theoretischen Perspektiven und der beobachteten Realität", verliert aber auf der anderen Seite die „Parsimonie“ allgemeiner Theorien (ebd.: 74). Schließlich sei nicht Abstraktion und maximale Vereinfachung das Ziel des Akteurzentrierten Institutionalismus, ,sondern Konkretisierung und hinreichende Komplexität der Erklärung “ (Mayntz 2002: 13). 
Der Akteurzentrierte Institutionalismus zielt auf die kausale Rekonstruktion sozialer Makrophänomene, wobei eine „Abkehr vom empiriefernen Bau theoretischer Modelle zugunsten eines intensiven Interesses am verstehenden Nachvollzug sozialer (einschließlich politischer und ökonomischer) Entwicklungen und Ereignisse" angestrebt wird (Mayntz 2009: 83). Unter kausaler Rekonstruktion wird die Erklärung des fraglichen Makrophänomens durch die Identifikation der für sein Zustandekommen beteiligten Prozesse und Wechselwirkungen verstanden. Dabei dient insbesondere die Erfassung relevanter situativer Gegebenheiten und Handlungen von (individuellen und komplexen) Akteur*innen als empirische Grundlage (ebd.: 85). Es handelt sich also um eine Verknüpfung von Mikro- und Makroperspektive, indem bei der kausalen Rekonstruktion von Makrophänomenen eine Mikrofundierung unter Berücksichtigung struktureller bzw. institutioneller Faktoren angestrebt wird.

Als wesentliche Triebkräfte des sozialen Geschehens und damit besonders erklärungskräftige Faktoren macht der Akteurzentrierte Institutionalismus Interessen verfolgende Akteur*innen auf der einen und Institutionen auf der anderen Seite aus (Mayntz 2009: 83). Als Akteur*innenkategorien werden Individuen, aggregierte Akteur*innen und komplexe Akteur*innen konzeptualisiert. Ein Akteur*innen-Aggregat ist eine forschungspragmatische Zusammenfassung von Individuen, die angesichts ähnlicher Präferenzen parallel zueinander gleichgerichtet handeln (bspw. akademischer Mittelbau) (Scharpf 2006: 98-100). Davon zu unterscheiden sind komplexe Akteur*innen, innerhalb derer die beteiligten Individuen koordiniert handeln und durch die als Resultat interner Interaktionen eine gemeinsame Wirkung erzeugt wird. In der Kategorie der komplexen Akteur*innen lassen sich wiederum kollektive und korporative Akteur*innen voneinander abgrenzen: Während kollektive Akteur*innen von den Präferenzen ihrer Mitglieder abhängig sind und von diesen kontrolliert werden (bspw. wissenschaftliche Fachgemeinschaften, Fakultäten, Arbeitskreise), sind korporative Akteur*innen typischerweise top-down organisiert (bspw. Ministerien, Ressortforschungseinrichtungen) (Scharpf 2006: 101-107; Schrape 2012: 3-4).

Akteur*innen handeln auf Basis ihrer subjektiv wahrgenommenen Realität intentional (Scharpf 2006: 47-48). Sie verfügen über Handlungsressourcen, die es ihnen ermöglichen, ein Ergebnis in bestimmter Hinsicht zu beeinflussen. Handlungsressourcen umfassen persönliche Merkmale (Intelligenz, Human- und Sozialkapital), materielle Ressourcen (Geld, technische Ausstattung) und privilegierten Informationszugang. Ebenfalls zu den Handlungsressourcen zählen institutionelle Regeln, die den Akteur*innen Kompetenzen und Rechte zuweisen (ebd.: 86). Handlungsorientierungen sind charakteristische Wahrnehmungen und Präferenzen der Akteur*innen, die durch den institutionellen Kontext beeinflusst 
sind. Sie werden durch den Stimulus eines Problems aktiviert und sie bestimmen die Bewertung des Status quo sowie die Wirksamkeit und Wünschbarkeit möglicher Handlungsoptionen (ebd.: 86-87). Es lassen sich kognitive von inhaltlichen und relationalen Aspekten der Handlungsorientierung unterscheiden (Mayntz und Scharpf 1995: 53-57):

„Kognitive Orientierungen betreffen die Wahrnehmung der Handlungssituation und ihrer kausalen Struktur, der verfügbaren Handlungsoptionen und erwartbaren Ergebnisse. Ihre Bedeutung für den Handlungserfolg der Akteure liegt ebenso auf der Hand wie die Schwierigkeit ihrer empirischen Ermittlung [.]“ (Mayntz und Scharpf 1995:

53)

Die motivationalen Aspekte der Orientierung sind die eigentlichen „Antriebsfaktoren für ein sinnhaftes Handeln“ oder auch "Auswahlgesichtspunkte bei der Wahl zwischen Handlungsoptionen" (Mayntz und Scharpf 1995: 54). Sie leiten sich aus den handlungsleitenden Interessen, normativen Erwartungen und Identitätskonstruktionen des Akteurs bzw. der Akteurin ab und lassen sich nicht akteur*innen- und situationsübergreifend gewichten. Ein weiterer, selten hervorgehobener, Aspekt der Handlungsorientierung ist die Interaktionsorientierung, die auf der Interpretation der Beziehung zwischen mehreren Akteur*innen beruht. Gemäß Mayntz und Scharpf (1995: 57) definieren die Interaktionsorientierungen die Beziehung ,entweder als eine ,feindliche‘, in der der Verlust des anderen als eigener Gewinn erscheint, eine ,kompetitive', in der es um die Differenz zwischen eigenem und fremdem Gewinn geht, eine ,egoistisch-rationale', in der allein der eigene Gewinn zählt, und eine ,kooperative‘, in der das Streben nach gemeinsamem Nutzen dominiert".

Entscheidungen werden selten durch eine*n isolierte*n Akteur*in gefällt, sondern sind in der Regel das Resultat von Interaktionen, innerhalb derer neben Akteur*innen auch „Konstellationen“ und „Interaktionsformen“ analytisch unterschieden werden. Konstellation beschreibt die Kombination beteiligter Akteur*innen, ihre Handlungsoptionen und Präferenzen in Bezug auf das gewünschte Ergebnis. Interaktionsform steht für den Modus der Entscheidungsfindung. Mayntz und Scharpf (1995: 61-62) wählen hier als abstrahierte Grundformen sozialer Handlungskoordination die einseitige und wechselseitige Anpassung, Verhandlungen, die Mehrheitsentscheidung sowie die hierarchische Steuerung. Das dieser Systematisierung zugrunde liegende Unterscheidungskriterium ist das Maß der individuellen Autonomie der Akteur*innen in Bezug auf die Durchsetzung der eigenen Präferenzen im Enscheidungsprozess: 
„Am einen Ende der Skala kommt eine Koordination nur in der Weise zustande, daß die einzelnen Akteure sich je für sich (und sogar ohne Kenntnis der Interdependenz ihrer Handlungen) an die von anderen beeinflußten Bedingungen ihrer Handlungssituation anpassen [...]. Bei wechselseitiger Anpassung [...], der nächsten Stufe, handeln die Akteure zwar weiterhin je für sich, nun aber in Kenntnis ihrer Interdependenz und in rationaler Antizipation der Aktionen und Reaktionen ihrer Partner oder Gegner. [...] Auf der folgenden Stufe, die der Govemance-Form des ,Marktes ' entspricht, kommt es zu expliziten, aber sachlich und zeitlich begrenzten und typischerweise bilateralen Vereinbarungen zwischen den Akteuren. ,Netzwerke'können demgegenüber als auf größere Dauer angelegte Verhandlungssysteme charakterisiert werden. " (Mayntz und Scharpf 1995: 61)

Hinzu kommen Abstimmungen, bei denen verbindliche Entscheidungen kollektiv durch Mehrheitsvotum getroffen werden. Wenn nicht nur die Verfügung über kollektivierte Handlungsressourcen, sondern auch die Willensbildung einer allenfalls auf diffuse Unterstützung angewiesenen - Autorität übertragen wird, fällt dies unter hierarchische Steuerung. Wichtig ist hierbei die Fähigkeit, Entscheidungen auch gegen den Willen der anderen Beteiligten treffen zu können und die eigenen Präferenzen ohne Kompensation oder Verhandlung durchsetzen zu können (Mayntz und Scharpf 1995: 62).

Akteur*innen, Konstellationen und Interaktionsformen sind beeinflusst durch den institutionellen Kontext. Er prägt Akteur*innen, indem er Handlungsressourcen und Handlungsorientierungen sowie die innere Organisation komplexer Akteur*innen strukturiert, er konstituiert Akteur*innenkonstellationen, indem er beteiligte Akteur*innen sowie deren mögliche Handlungsoptionen benennt und mit Anreizen versieht, und er bestimmt Interaktionsformen, indem er Entscheidungsregeln vorgibt (siehe Abb. 5.1) (Treib 2015: 280). Der Akteurzentrierte Institutionalismus erhebt Institutionen zur wichtigsten Einflussgröße auf - und deshalb nützlichsten Informationsquelle über - Entscheidungsprozesse, weil sie nicht nur für die beforschten Akteur*innen eine wichtige Handlungsorientierung in komplexen Umgebungen bereitstellen, sondern auch im Normalfall zum gemeinsamen Wissen aller Akteur*innen gehören und daher auch dem*der Forschenden relativ leicht zugänglich sein sollten (Scharpf 2006: 78). Zur Rekonstruktion von Makrophänomenen wird daher zunächst mit solchen allgemein zugänglichen Institutionen operiert, bevor, wo nötig, auf die schwerer zugänglichen individuellen Faktoren rekurriert wird (Treib 2015: 282; Scharpf 2006: 111-114).

Im Akteurzentrierten Institutionalismus geben Institutionen Handlungsoptionen vor und setzen dem Handeln zugleich Grenzen, ohne es jedoch zu 


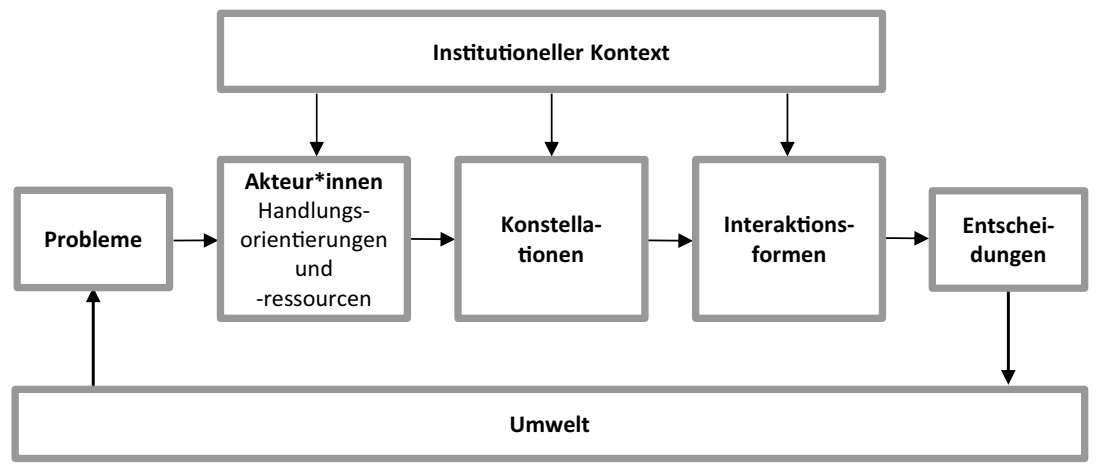

Abb. 5.1 Das analytische Modell des Akteurzentrierten Institutionalismus (Eigene Darstellung nach Scharpf (2006: 83))

determinieren (Mayntz 2009: 83). Institutionen bilden somit einen stimulierenden, ermöglichenden, aber auch restringierenden Handlungskontext (Mayntz und Scharpf 1995: 43). Der institutionelle Kontext definiert die Regeln,

\begin{abstract}
„[...] deren Einhaltung man von anderen erwarten kann und sich selbst zumuten lassen ти $\beta$, konstituiert Akteure und Akteurkonstellationen, strukturiert ihre Verfügung über Handlungsressourcen, beeinflußt ihre Handlungsorientierungen und prägt wichtige Aspekte der jeweiligen Handlungssituation, mit der der einzelne Akteur sich konfrontiert sieht. Der institutionelle Rahmen umschließt jedoch nicht alle Arten von Handlungen und handlungsrelevanten Faktoren, und er bestimmt auch dort, wo er gilt, Handlungen nicht vollständig. “ (Mayntz et al. 1995: 49)
\end{abstract}

Institutionen umfassen nicht nur formelle Regeln, deren Einhaltung durch das Rechtssystem, den Staatsapparat oder übergeordnete Organisationsformen auf Grundlage formalisierter Verfahren und Instrumente überwacht wird, sondern auch informelle Normen, die von den Akteur*innen ,im Allgemeinen beachtet werden und deren Verletzung durch Reputationsverlust, soziale Mißbilligung, Entzug von Kooperation und Belohnung oder sogar durch soziale Ächtung“ seitens des sozialen Umfelds sanktioniert wird (Scharpf 2006: 77). Indem Institutionen die zur Verfügung stehenden Handlungen mit positiven und negativen Anreizen verbinden, bestimmen sie die Wahrscheinlichkeit, nach der sich rationale, nutzenmaximierende Akteur*innen für diese Alternativen entscheiden werden (ebd.: 78). In Abgrenzung zu einigen Ausprägungen des soziologischen 
Neo-Institutionalismus, fallen im Akteurzentrierten Institutionalismus handlungsprägende kognitive Elemente und unhinterfragte Praktiken des Alltagslebens nicht unter den Institutionenbegriff. Stattdessen wird auf Regeln und Normen fokussiert, wodurch zum einen die Handlungsspielräume der beobachteten Akteur*innen akzentuiert werden und zum anderen klargestellt wird, dass Institutionen das Ergebnis absichtsvollen Handelns von Akteur*innen sein können (Mayntz et al. 1995: 45-46; Schrape 2012). Das Konzept der Institution ist im Akteurzentrierten Institutionalismus auf Regelsysteme beschränkt. Soziale Entitäten werden also nicht als Institution deklariert, sondern stattdessen bspw. als ,Organisation' oder , korporative*r Akteur*in' (Scharpf 2006: 77). Im Akteurzentrierten Institutionalismus werden nicht alle möglichen Institutionen, auf deren Grundlage Akteur*innen agieren, als institutioneller Kontext erfasst, sondern die wichtigsten Einflüsse auf jene Akteur*innen und Interaktionen, die wesentlich für die Erklärung des Makrophänomens sind (ebd.: 78).

\subsection{Der institutionelle Kontext der Wissenschaft}

Die Wissenschaft wird in dieser Arbeit als ein soziales System betrachtet, in welchem Wissenschaftler*innen begründete und für gesichert erachtete Erkenntnisse in einem fachgemeinschaftlichen Forschungsprozess und auf der Basis eines geteilten Wissensstandes gewinnen. Sie tun dies, indem sie die Erkenntnisse ihrer Fachkolleg*innen beobachten, prüfen und darauf aufbauend selbst Erkenntnisse produzieren und veröffentlichen, womit sie wiederum den gemeinsamen Wissensstand verändern. Die einzelnen Elemente dieser Definition werden im Folgenden beschrieben. Dabei werden die in Kapitel 4 dargelegten Mechanismen und institutionellen Einflussgrößen der Wissenschaft auf Basis des in Abschnitt 5.2 dargelegten Ansatzes des Akteurzentrierten Institutionalismus zusammengefasst.

Die wissenschaftliche Gemeinschaft, hier verstanden als die Gesamtheit aller wissenschaftlichen Akteur*innen, ist in Fachgemeinschaften untergliedert. Fachgemeinschaften gruppieren sich um spezifische Forschungsgegenstände, Methoden oder Theorien und kommunizieren über diese miteinander organisations- und generationenübergreifend in wissenschaftlichen Fachbeiträgen. Sie heben sich hervor als hinreichend homogene, dynamische Kommunikationszusammenhänge von Akteur*innen, in denen kollektiv ein jeweils spezifisches Wissen produziert wird. Als solche lassen sie sich auf unterschiedlichen Maßstabsebenen bspw. über Verdichtungen im Ko-Autorenschafts- oder Zitationsnetzwerk identifizieren. Indem Fachgemeinschaften entlang der fortlaufenden Weiterentwicklung eines spezifischen Wissensstandes emergieren, ist diese Weiterentwicklung gleichzeitig 
die Grundlage für die Neuentstehung, die Metamorphose und das Aussterben von Fachgemeinschaften. Die gegenstandsbezogenen, systematischen Erörterungen von Fachgemeinschaften werden auch als Diskurse bezeichnet. Ein*e Akteur*in ist in der Regel polythematisch ausgerichtet, das heißt, er nimmt an mehreren Diskursen teil und lässt sich mehreren Fachgemeinschaften zuordnen.

Fachgemeinschaften sind grundsätzlich unabhängig vom Grad ihrer formellen Institutionalisierung zu denken, was nicht ausschließt, dass sie in formalisierten Organisationszusammenhängen repräsentiert sind. Dementsprechend wird die Mitgliedschaft zu einer Fachgemeinschaft nicht durch die Entscheidung einer zentralen Instanz erworben, sondern dadurch, dass ein*e Akteur*in einen eigenen Beitrag zum geteilten Wissen hinzufügt und dieser Beitrag von den Mitgliedern der Gemeinschaft rezipiert wird. Von Fachgemeinschaften konzeptionell zu unterscheiden sind Disziplinen, die zwar auch Kommunikationsstrukturen darstellen, die aber stets auch formell institutionalisiert sind, bspw. in Form von disziplinspezifischen Karrierestrukturen und Lehrbüchern. Die Wissensproduktion einer Disziplin oder Fachgemeinschaft ist durch ihr Maß an attention space repräsentiert und beeinflusst, also der Anzahl wissenschaftlicher Beiträge, die veröffentlicht und rezipiert werden können.

Wissenschaftliches Wissen wird definiert als innerhalb des wissenschaftlichen Systems verhandelte Erkenntnisse in Form von Theorien, Modellen, Methoden, Beobachtungen, Fakten oder Normen. Konstitutiv ist dabei, dass das Wissen von mindestens einem Mitglied einer wissenschaftlichen Gemeinschaft öffentlich formuliert und von mindestens einem anderen Mitglied anerkannt und für die weitere Erkenntnisproduktion eingesetzt wird. Das wissenschaftliche, fachgemeinschaftliche oder auch individuelle Wissen lässt sich als diachrone Wissensstruktur begreifen, also als ein Netzwerk aus aufeinander aufbauenden Forschungsprozessen und Erkenntnissen bzw. Forschungspfaden. Die Gültigkeit und Relevanz wissenschaftlichen Wissens ist sozial konstruiert und wird durch die wiederholte wissenschaftliche Kommunikation darüber (re)produziert. Auch das Wissen selbst ist ein soziales Produkt, indem Wissenschaftler*innen stets auf die Werke anderer Wissenschaftler*innen rekurrieren und ihre Forschungen und Argumentationen (zustimmend oder ablehnend) auf diese stützen. Die Erweiterung und Beobachtung wissenschaftlichen Wissens erfolgt also im Kollektiv: Welche Forschungsprobleme Priorität haben, welche Erkenntnisse wahr sind und welche wissenschaftlichen Handlungen und Akteur*innen belohnt oder sanktioniert werden, entscheidet weitgehend die wissenschaftliche Gemeinschaft in einem dezentralen und diffusen Prozess, in dem die Einzelentscheidungen ihrer Mitglieder in einen Gesamtzusammenhang integriert werden. 
Das veröffentlichte und von der wissenschaftlichen Gemeinschaft rezipierte Wissen wird als Fachbeitrag gefasst. Die wissenschaftliche Publikation ist hierfür das maßgebliche Medium, also die Veröffentlichung wissenschaftlicher Ergebnisse in einer Monografie, als Beitrag in einem Sammelband, als Aufsatz in einer wissenschaftlichen Fachzeitschrift oder als graue Literatur. In wissenschaftlichen Publikationen rekurrieren die Autor*innen stets auf die Werke anderer Wissenschaftler*innen und machen dies durch Zitationen explizit. Dabei lassen sich zwei Funktionen der Zitation unterscheiden: die formale Funktion (Offenlegung der Quellen) und die latente Funktion (Verteilung und Entzug von Reputation). Aus der formalen Funktion ergibt sich das Verständnis einer Zitation als textliche Spur und somit sichtbares Element eines diachronen Wissensnetzwerks. Die latente Funktion bezieht sich auf die durch das Zitieren und Nicht-Zitieren vermittelte Belohnung und Sanktionierung der Fachkolleg*innen.

Auch wenn sich die wissenschaftliche Relevanz eines Fachbeitrags erst Jahre nach seiner Veröffentlichung zeigt, lässt sich eine Reihe von Faktoren identifizieren, die - im Sinne der Nachrichtenwerttheorie in den Medien- und Kommunikationswissenschaften (siehe Kp. 3.3) - eine hohe Aufmerksamkeit gegenüber einem Beitrag wahrscheinlicher machen. Dabei ist zunächst entscheidend, dass die Erkenntnis seitens der Fachgemeinschaft für wahr und originell befunden wird und dass sie für eine möglichst große Zahl an Forscher*innen inhaltlich passfähig im Rahmen ihrer jeweiligen Forschung ist. Um überhaupt eine kritische Zahl an Rezipient*innen zu gewinnen, sollte die produzierte Erkenntnis in einem Medium mit einer möglichst großen Reichweite erscheinen. Für die Reichweite des Mediums sind (im Falle von Zeitschriften, Reihen oder Sammelbänden) insbesondere dessen Reputation, Sprache und inhaltlicher Zuschnitt entscheidend. Darüber hinaus spielen für die fachgemeinschaftliche Verwendung einer Erkenntnis soziale und strategische Kriterien eine Rolle, etwa dadurch, dass besonders bekannte Autor*innen und Publikationen bevorzugt rezipiert und zitiert werden, weil deren Erwähnung seitens der Leser*innenschaft belohnt wird (Matthäus Effekt, siehe Kp. 4.2.2).

Die Relevanz eines Fachbeitrags manifestiert sich im Rahmen der nachfolgenden Wissensproduktion in der Qualität und Frequenz seiner - durch Zitierungen dokumentierten - Verwendung durch die Fachgemeinschaft. Die Urheberschaft relevanter Erkenntnisse ist das wesentliche Kriterium für die Zuschreibung von Reputation. Indem die Mitglieder der Fachgemeinschaft um Reputation konkurrieren, gleichzeitig aber deren Zuschreibung selbst verantworten, wird die Herstellung von Relevanz zu einem über epistemische Mechanismen hinausgehenden sozialen und politischen Prozess. Gleichzeitig ist diese Selbstreferenzialität Grundlage für die häufig hervorgehobene Autonomie der Wissenschaft. 
Diese interessiert sich für Entwicklungen und Informationen aus anderen Teilbereichen der Gesellschaft nur insofern, als dass sie für die Produktion relevanten wissenschaftlichen Wissens Bedeutsamkeit erlangt.

Das System Wissenschaft ist auf vielfältige Weise und in zunehmenden Maße mit der gesellschaftlichen Umwelt strukturell gekoppelt. Für die Herstellung ihrer Legitimität sowie die Bereitstellung von Infrastrukturen und finanziellen Ressourcen ist die Wissenschaft auf die Leistungen anderer gesellschaftlicher Teilsysteme angewiesen. Diese Abhängigkeit kann als „Einfallstor“ (Aljets 2015: 77) für verschiedenartige Beeinflussungsversuche durch nicht-wissenschaftliche Akteur*innen gesehen werden. Insbesondere im Rahmen von Auftragsforschungen, politisch beeinflusster Fördermittelvergabe oder Ressortforschung modifizieren Politik, Verwaltung oder Wirtschaft die wissenschaftliche Wissensproduktion auf der Basis ihrer jeweiligen Nutzenerwartungen und verändern die wissenschaftliche Sozialstruktur sowie die inhaltliche Schwerpunktsetzung unabhängig von wissenschaftsinternen Reputationsmechanismen. Auch die Aufmerksamkeitskriterien der Öffentlichkeit beeinflussen die wissenschaftliche Wissensproduktion, indem Wissenschaftler*innen etwa über die Herstellung gesamtgesellschaftlicher Aufmerksamkeit politisch gesteuerte Ressourcenzuweisungen zu beeinflussen suchen. Die extern vermittelten Selektionsprinzipien haben wiederum Rückwirkungen auf die Ressourcenallokation wissenschaftlicher Akteur*innen und folglich auf die Wissensproduktion. Da sich die verschiedenen Funktionssysteme der Gesellschaft ständig wandeln, verändern sich auch die von außen an die Wissenschaft herangetragenen Prioritäten und Beurteilungskriterien. Durch verschiedene Kanäle vermittelt, ergibt sich ein Interdependenzverhältnis, das wesentlich zum institutionellen Kontext der Wissenschaft beiträgt. In dem Maße, wie die Wissenschaft auf die sich verändernden externen Erwartungen reagiert, wird die gesellschaftliche Umwelt zur treibenden Kraft für den innerwissenschaftlichen Wandel. In Bezug auf die Entwicklung der Aufmerksamkeit gegenüber bestimmten Themen stellt sich an dieser Stelle die Frage, inwiefern die wissenschaftsintern verhandelten Forschungsbedarfe und die fachöffentliche Aufmerksamkeitsverteilung durch wissenschaftsexterne Nutzenerwartungen und Relevanzkriterien beeinflusst werden.

Die Mechanismen der kollektiven Wissensproduktion in der Fachgemeinschaft zusammen mit wissenschaftsexternen Kanälen der Einflussnahme bilden den institutionellen Kontext der Wissenschaft. Dieser prägt wissenschaftliche Akteur*innen, indem er Handlungsressourcen und Handlungsorientierungen sowie die innere Organisation komplexer Akteur*innen strukturiert. Er konstituiert Entscheidungssituationen, indem er Entscheidungsregeln vorgibt, die beteiligten Akteur*innen benennt, ihnen Handlungsoptionen vorgibt und diese 
mit Anreizen versieht. Wissenschaftsexterne Akteur*innen und Strukturen nehmen auf der einen Seite direkt Einfluss auf die Entscheidungen wissenschaftlicher Akteur*innen bezüglich des Forschungsinhalts (bspw. mit Forschungsaufträgen), schaffen und modifizieren auf der anderen Seite aber auch die Rahmenbedingungen des Wissenschaftssystems (bspw. durch das Personalrecht) und beeinflussen somit indirekt - vermittelt durch die fachgemeinschaftliche Wissensproduktion - die den Forschungsinhalt betreffenden Entscheidungen wissenschaftlicher Akteur*innen.

\subsection{Handlungsressourcen und Handlungsziele wissenschaftlicher Akteur*innen}

Als wissenschaftliche Akteur*innen sind in dieser Arbeit in erster Linie Wissenschaftler*innen zu fassen, also Individuen, die über einen Hochschulabschluss verfügen und mittels eigener Beiträge an der wissenschaftlichen Kommunikation teilnehmen. Sie sind in der Regel an Hochschulen, außeruniversitären Forschungsorganisationen oder anderen Einrichtungen mit Forschungsausrichtung beschäftigt oder organisationell angebunden. Auch kollektive Akteur*innen (bspw. Fakultäten, Leibnitz-Forschungsinstitute) werden als wissenschaftliche Akteur*innen gefasst, während korporative Akteur*innen, zumindest im Rahmen dieser Forschung, zwar Einfluss auf die Wissenschaft nehmen (bspw. Ministerien), aber nicht in der Wissenschaft zu verorten sind.

Wissenschaftliche Akteur*innen handeln auf Basis ihrer subjektiv wahrgenommenen Realität intentional. Sie verfügen über Handlungsressourcen, die es ihnen ermöglichen, ein Ergebnis in bestimmter Hinsicht zu beeinflussen, und sie agieren auf Basis von Handlungsorientierungen, also charakteristischen Wahrnehmungen und Präferenzen. Die Ressourcen und Orientierungen wissenschaftlichen Handelns werden im Folgenden systematisiert und erläutert.

Während institutionalistische Ansätze bislang auf die sozialen Anreizstrukturen fokussieren, und dabei insbesondere die formale Koordination, die Reputation und die materiellen Ressourcen als Einflussfaktoren oder Kapitalarten unterscheiden (siehe Kp. 4.3), wird die Rolle epistemischer Faktoren in der Regel nicht oder nur in nachgeordneter Funktion, als Mittel zur Erlangung von Reputation, konzeptualisiert. Dies ist mit der jeweils beabsichtigten Betonung der Rolle eben jener sozialen Faktoren zu erklären, mit Hilfe derer (trans-epistemische) Einflussnahmen auf die Forschung sowie die Interdependenzen wissenschaftlicher Akteur*innen analytisch gefasst werden können. Das Erkenntnisinteresse dieser Arbeit ist jedoch anders gelagert. Epistemische Faktoren sollen hier sehr 
wohl konzeptualisiert werden, da die Entstehung von Themen in der Wissenschaft im Fokus der Analyse steht. Im Sinne der Mikrofundierung von Makrophänomenen lässt sich die auf die Akteur*innenebene heruntergebrochene Fragestellung formulieren als, Wie wählen wissenschaftliche Akteur*innen ein Thema?'. Die bisher persönlich oder fachgemeinschaftlich angeeignete Kompetenz, also die Fertigkeiten und das Wissen, ist ein zentraler Einflussfaktor für zukünftige Forschungsentscheidungen und insbesondere für die Auswahl von Forschungsproblemen, wie in Abschnitt 4.2.3 im Rahmen der Erläuterung epistemischer Mechanismen der Wissensproduktion hergeleitet werden konnte. Hier sind es insbesondere die individuellen Einschätzungen fachgemeinschaftlicher Forschungsbedarfe sowie der eigenen Kompetenzen im Vergleich mit den Fachkolleg*innen, die für die Wahl von Forschungsproblemen ausschlaggebend sind. Der Akteurzentrierte Institutionalismus kommt für politische Entscheidungen zu einer ähnlichen Einschätzung, indem er Handlungsressourcen von Akteur*innen konzeptionell als entscheidungsrelevant erfasst und darunter neben Sozialkapital und materiellen Ressourcen auch Humankapital, also das Wissen und die Fähigkeiten von Akteur*innen, miteinbezieht.

Kompetenz lässt sich aber nicht nur als Handlungsressource, sondern dessen Erweiterung auch als Handlungsorientierung konzeptualisieren. Solcherlei Wahrnehmungen und Präferenzen leiten sich gemäß Scharpf und Mayntz aus den Interessen, Erwartungen und Identitätskonstruktionen der Akteur*innen ab und lassen sich nicht akteur- und situationsübergreifend gewichten. Häufig wird das Streben nach neuem und wahrem Wissen als Mittel zum Zweck eingestuft, das heißt, das Aufbauen neuen Wissens dient allein einem anderen, sozialen Handlungsziel, nämlich zumeist der Erhöhung der Reputation. Demgegenüber hebt Maton (2003: 61) die Rolle nicht-sozialer Motivationen in der Wissenschaft hervor. Er räumt zwar auf der Grundlage von Bourdieus soziologischer Theorie die große Bedeutung sozial orientierter Motivationen (,,social interestedness “) für Wissenschaftler*innen ein, stellt aber gleichzeitig klar, wissenschaftliches Engagement sei „mehr als das“. Das Forschen, Vortragen, Verfassen und Lesen von Publikationen sei nicht immer allein mit dem Streben nach sozialer Autorität zu erklären, sondern eben auch mit dem intrinsischen Wunsch, Forschungsprobleme zu lösen, bzw. mit dem unvermittelten Streben nach neuen Erkenntnissen. Maton (2003: 62) schlägt daher vor, „epistemisches Kapital“ als Handlungsorientierung zu konzeptualisieren:

„I suggest we can add [...] the concept of epistemic capital, the ability to better explain the (social) world. This captures the way in which actors within the intellectual field 
engage in strategies aimed at maximising not merely resources and status but also epistemic profits, that is, better knowledge of the world. "

Dieser Konzeptualisierung der Wissensorientierung als epistemisches Kapital schließt sich diese Arbeit an, allerdings (1.) mit expliziter Berücksichtigung der sozialen Dimension dieses Wissens und (2.) ergänzt um die Fertigkeiten, die ein*e Akteur*in erwerben kann (s. u.). Darüber hinaus werden mit leichten Anpassungen die Kategorien aus Abschnitt 4.3 übernommen. Es werden also vier grundlegende Kapitalsorten unterschieden, die einerseits als Handlungsressource dienen, deren Erwerb aber andererseits auch eine Handlungsorientierung für wissenschaftliche Akteur*innen darstellen: epistemisches Kapital, soziales Kapital, ökonomisches Kapital und politisches Kapital. Zusammengenommen werden die vier genannten Handlungsressourcen auch als wissenschaftliches Kapital wissenschaftlicher Akteur*innen bezeichnet.

Unter epistemischem Kapital oder auch fachlich-sozialer Kompetenz werden in dieser Arbeit das Wissen und die Fertigkeiten wissenschaftlicher Akteur*innen verstanden, die benötigt werden, um bspw. einen Fachbeitrag oder einen Drittmittelantrag zu erstellen und erfolgreich in die kollektive Wissensproduktion einzubringen. Das Wissen beinhaltet ein Verständnis von Inhalten und Methoden (etwa ,wo besteht Forschungsbedarf?'), Akteur*innen (etwa ,wer hat sich durch einschlägige Forschung besonders hervorgetan?'), Medien (etwa ,in welchen Fachzeitschriften lässt sich ein Fachbeitrag möglichst effektiv oder schnell unterbringen?') usw. Unter Fertigkeiten (oder: skills) wird das Vermögen der Akteurin bzw. des Akteurs gefasst, bestimmte Aufgaben der Forschung - etwa das Verfassen eines Fachbeitrags oder die Anwendung einer spezifischen Erhebungsmethode - effektiv erfüllen zu können. Das epistemische Kapital hat neben der fachlichen immer auch eine soziale Seite. Dies folgert aus dem Verständnis dieser Arbeit, dass wissenschaftliches Wissen (und dessen Geltung) immer sozial konstruiert ist, sodass Fachwissen (etwa Theorieverständnis) und Sozialwissen (etwa Wissen um Reputationsverteilung oder Machtbeziehungen) nicht voneinander zu trennen sind, sondern ein und dasselbe Wissen beschreiben. Schließlich gehört zum Theorieverständnis auch ein Wissen darüber, welche Theorien aktuell in der Fachgemeinschaft als bekannt vorausgesetzt werden können und welche dort als besonders vertrauenswürdig und nützlich angesehen sind. Ebenso ist die Begründung und Erläuterung des Forschungsbedarfs nicht allein durch logische Herleitung zu leisten, sondern impliziert ein Verständnis bspw. über aktuelle Relevanzkriterien des Zielpublikums bzw. der Fachgemeinschaft. Auch Fertigkeiten haben nicht nur eine fachliche Komponente (bspw. das Beherrschen einer Statistik-Anwendung), sondern auch eine soziale (bspw. kommunikative 
Fertigkeiten). Fertigkeiten und Wissen sind interdependent, indem etwa taktisches Geschick ein Mindestmaß an instrumentellen Wissen erfordert oder indem bestimmte Fertigkeiten eingesetzt werden müssen, um Wissen zu erwerben.

$\mathrm{Zu}$ den Handlungsressourcen zählt weiterhin das soziale Kapital, also gemäß Bourdieu (1983: 190) „die Gesamtheit der aktuellen und potentiellen Ressourcen, die mit dem Besitz eines dauerhaften Netzes von mehr oder weniger institutionalisierten Beziehungen gegenseitigen Kennens oder Anerkennens verbunden sind". Bezogen auf einzelne (individuelle oder komplexe) wissenschaftliche Akteur*innen beschreibt Sozialkapital den ihnen zu Gute kommenden vorteilhaften Effekt der Netzwerkstruktur, der darin besteht, diesen „,breitere Handlungsmöglichkeiten oder Zugang zu Ressourcen zu eröffnen " (Jansen und Diaz-Bone 2014: 73). Als Teilaspekte des sozialen Kapitals werden wissenschaftliche Reputation und persönliche Beziehungen voneinander unterschieden. Die Reputation (bzw. soziale Anerkennung, Prestige) ist auch für Außenstehende auf der Grundlage formeller Indikatoren in gewissem Maße erkennbar und wird auch abseits persönlicher Bekanntschaften dezentral durch die Fachkolleg*innen zugeschrieben. Sie manifestiert sich bspw. in Zitationsindizes, in Beteiligungen in Forschungsprojekten, in organisationalen Zugehörigkeiten oder in durchlaufenen Karrierestationen. Persönliche Beziehungen, etwa zu Fachkolleg*innen, Herausgeber*innen, Gutachter*innen oder Fördermittelgeber*innen, entstehen durch den direkten Kontakt und senken die Transaktionskosten wissenschaftlicher Austauschbeziehungen, wie bspw. gemeinsamer Projektanbahnungen, Ko-Autorenschaften oder der Weitergabe von (exklusiven) Informationen. Zwar werden die beiden Teilaspekte - Reputation und soziale Beziehungen - konzeptionell unterschieden, sie lassen sich aber nicht gänzlich voneinander trennen. So ist bspw. die Zuschreibung wissenschaftlicher Anerkennung seitens befreundeter Fachkolleg*innen mitunter eine wichtigere Handlungsmotivation für Wissenschaftler*innen als die in Zitaten und Positionen geronnene Reputationsallokation seitens der erweiterten Fachgemeinschaft. Persönliche Beziehungen beeinflussen zudem den Reputationserwerb, etwa wenn Zitationen nicht allein aus inhaltlichen Faktoren oder Ko-Autorenschaften nicht allein aus der tatsächlichen Mitwirkung resultieren, sondern auch aus Freundschaftsbeziehungen. Genauso ist es umgekehrt für Akteur*innen mit höherer Reputation leichter, persönliche Beziehungen aufzubauen, weil seitens der Fachkolleg*innen ein gesteigertes Interesse an der Relation besteht.

Das ökonomische Kapital umfasst die Geldmittel, die Ausstattung und das Personal eines Akteurs bzw. einer Akteurin und wird auch als materielle Ressourcen gefasst. Zu den Geldmitteln zählen das persönliche Einkommen sowie finanzielle Ressourcen, die in unterschiedlichem Ausmaß zweckgebunden sein können. 
Die Ausstattung umfasst die Verfügbarkeit oder den Besitz von technischen Gerätschaften, Räumen, Literatur und Daten. Personal steht für die personellen Ressourcen oder Mitarbeiter*innen, für die der*die Akteur*in - in unterschiedlichem Maße - weisungsbefugt ist. Materielle Ressourcen in der Wissenschaft werden primär in Form von Grundmitteln und Drittmitteln zur Verfügung gestellt. Mit Grundmitteln werden die laufenden Ausgaben der Universitäten und Forschungsinstitute bezeichnet, wobei in dieser Arbeit der auf Forschung entfallene Teil der Sach- und Personalmittel interessiert. Der Begriff Drittmittel umfasst Gelder, die nicht direkt von der unterhaltenden Universität oder dem unterhaltenden Forschungsinstitut stammen, sondern (zusätzlich) von öffentlichen oder privaten Stellen bereitgestellt werden. Sie werden meist in einem kompetitiven Verfahren von einzelnen oder kooperierenden Forscher*innen, Lehrstühlen oder Instituten eingeworben. Werben wissenschaftliche Akteur*innen ein Drittmittelprojekt ein, so können sie mit einem Schlag ihre Ausstattung erheblich steigern. Auf der anderen Seite bieten die langfristiger angelegten Grundmittel für wissenschaftliche Akteur*innen eine höhere Planbarkeit, und sie sind weniger zweckgebunden. Materielle Ressourcen sind eine wichtige Voraussetzung, um Forschung betreiben und am Reputationswettbewerb teilnehmen zu können. Ihr Besitz verspricht Handlungsspielraum und Einfluss, etwa indem durch Investitionen in die eigenen Forschungsthemen diese gestärkt und somit die Forschungslandschaft gemäß der eigenen Präferenzen geprägt werden kann. Zudem kann gerade in Zeiten der Zunahme prekärer Beschäftigungsformen die Sicherung des Lebensunterhalts zum zentralen Kriterium für die Aufnahme einer bestimmten entlohnten wissenschaftlichen Tätigkeit werden.

Ebenfalls zu den Handlungsressourcen zählt das politische Kapital, das auch als formales Kapital, institutionelle Macht oder Einfluss geführt werden kann. Das politische Kapital umfasst die Rechte, Pflichten und Einflussmöglichkeiten, die einem*einer Akteur*in im Rahmen der formalen Koordination der Wissenschaft zugeschrieben werden und die der*die Akteur*in zu nutzen versteht. Sie leiten sich also aus Gesetzen und Vorschriften ab und werden unter anderem auf Basis von Posten (bspw. Dekan*in), Funktionen (bspw. Gutachter*in in einem Forschungsprogramm, Herausgeber*in einer Zeitschrift), akademischen Graden (bspw. Doktor*in) oder Mitgliedschaften (bspw. in einer Akademie) verliehen. $\mathrm{Zu}$ dem politischen Kapital wird auch das Durchsetzungsvermögen in kollektiven Entscheidungssituationen gezählt, für das zum einen Fertigkeiten (bspw. Verhandlungsgeschick, strategisch-taktisches Können) und zum anderen akkumuliertes Wissen (über politisch-historische Hintergründe, Präzedenzfälle, mögliche Handlungsalternativen) entscheidend sind. Das Verfügen über Einfluss ermöglicht es Akteur*innen, bestimmte Themen bei Verteilungsentscheidungen zu fördern 
bzw. zu verhindern, beispielsweise dann, wenn in universitären Gremien über die Denomination von Lehrstühlen oder im Rahmen von Begutachtungen über die Finanzierung von Forschungsprojekten entschieden wird. Nicht zuletzt in hierarchischen Machtbeziehungen werden thematische Ausrichtungen verhandelt, bspw. bei der Bestimmung des Inhalts gemeinsamer Veröffentlichungen und Forschungsanträge.

Auch außerhalb der Wissenschaft akquirierte Handlungsressourcen können in der Wissenschaft in Wert gesetzt werden, sofern sie sich in die Handlungslogik des Systems übertragen lassen. So kann beispielsweise Wissen über die politische Ökonomie der lokalen Stadtentwicklung, das im Rahmen von zivilgesellschaftlichem Engagement akquiriert worden ist, im Rahmen der wissenschaftlichen Wissensproduktion eingesetzt werden. Ebenfalls können persönliche Beziehungen mit Akteur*innen aus Politik oder Wirtschaft in der Wissenschaft von Nutzen sein. Indem wissenschaftliche Akteur*innen auch in anderen gesellschaftlichen Kontexten agieren, können auch außerwissenschaftliche Motive in wissenschaftlichen Entscheidungssituationen ins Gewicht fallen. Als Beispiele seien das Ablehnen eines ethisch bedenklichen Forschungsprojekts zur Sicherung des eigenen Ansehens in Familie und Freundeskreis sowie das Anstreben der Doktorwürde zur Verbesserung alleinig außerwissenschaftlicher Karrierechancen genannt.

Das wissenschaftliche Kapital lässt sich - mit unterschiedlicher Effektivität - für bestimmte Zwecke investieren und gegeneinander eintauschen. Wer beispielsweise. über ein hohes Maß an epistemischem Kapital verfügt, hat gute Voraussetzungen, um Reputation zu erwerben, mit der er*sie wiederum eine hochrangige Position erlangen und Fördermittel einwerben kann. Eine hochrangige Position verleiht wiederum Reputation sowie gleichzeitig politisches Kapital und leichteren Zugang zu ökonomischem Kapital und persönlichen Beziehungen. Aufbauend auf Latour und Woolgar (1982, 1986) lässt sich dieser Mechanismus der iterierenden Übersetzung verschiedener Kapitalsorten als credit cycle beschreiben (siehe Kp. 4.3.4). Aufgrund mangelnder Entsprechungen des englischen Begriffs credit wird das Investieren und Eintauschen der jeweiligen Handlungsressourcen zum Zwecke der Kapitalvermehrung hier als wissenschaftlicher Akkumulationszyklus bezeichnet. Allerdings ist anzumerken, dass jede Kapitalsorte sich gegen jede andere Kapitalsorte eintauschen lässt, der Zyklus also nicht allein in eine Richtung verläuft und mehrere Prozesse parallel stattfinden können. Zudem handelt es bei der zyklischen Kapitalakkumulation nicht um einen linearen, planbaren Prozess. Stattdessen kann Kapital auch wieder verloren gehen, eine getätigte Investition nicht den gewünschten Ertrag 
bringen oder eine Handlung zu einer nicht intendierten Kapitalvermehrung verhelfen. An dieser Stelle sei auch darauf hingewiesen, dass die Grenzen zwischen den jeweiligen Kapitalsorten nicht immer scharf verlaufen, sondern dass mitunter Überschneidungen vorkommen. Einige beispielhafte Tauschmechanismen zwischen den jeweiligen Handlungsressourcen sind in Abb. 5.2 dargestellt.

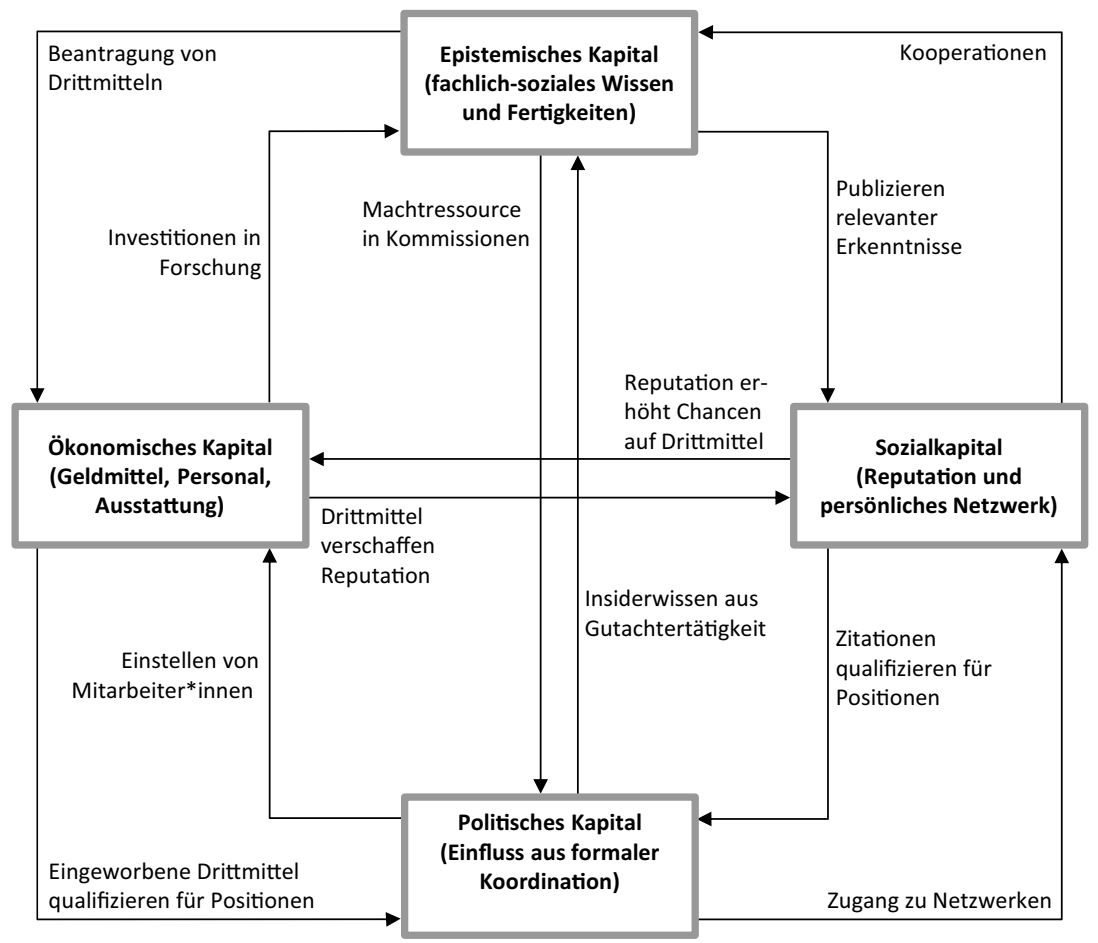

Abb.5.2 Kapitalakkumulationszyklus wissenschaftlicher Akteur*innen (Eigene Darstellung)

In der Erweiterung und Beschleunigung dieses Zyklus bzw. in der Vermehrung der Handlungsressourcen sind die Handlungsorientierungen wissenschaftlicher Akteur*innen zu erkennen. Insbesondere das Erlangen von Reputation wird als primäre Handlungsorientierung gefasst, durch das weitere Ziele, wie das Vermehren materieller Ressourcen, das Eingehen und die Pflege von Beziehungen oder 
das Erweitern des politischen Kapitals erreicht werden können. Wissenschaftliche Akteur*innen suchen also, die unterschiedlichen Kapitalsorten zu maximieren und treffen deshalb Entscheidungen, die diese Maximierung begünstigen. Durch Belohnung und Sanktionierung bzw. über die Gewährung und den Entzug der genannten Kapitalien werden die Forschungsentscheidungen wissenschaftlicher Akteur*innen steuerbar.

\subsection{Die wissenschaftliche Themenwahl}

Als nächster und letzter Baustein des analytischen Rahmens wird auf der Basis der vorangegangenen Kapitel die Entscheidungssituation skizziert, in der individuelle sowie komplexe wissenschaftliche Akteur*innen einen Forschungsgegenstand wählen. Demnach treffen wissenschaftliche Akteur*innen die Wahl ihrer Forschungsthemen (im Folgenden Themenwahl) in der Regel autonom auf der Grundlage ihrer persönlichen Interpretation (1.) der Entscheidungssituation, (2.) des institutionellen Kontextes (3.) ihrer Handlungsorientierungen und (4.) ihrer verfügbaren Handlungsressourcen. Diese einzelnen Faktoren und ihr Zusammenspiel im Zuge einer Themenwahl werden im Folgenden erläutert. Dabei wird primär die wissenschaftliche Einzelentscheidung in den Blick genommen, weil sie die häufigste und für die Themenentwicklung in einer Disziplin wichtigste Entscheidungssituation darstellt. Gemeint sind Entscheidungen, die ein*e Akteur*in - ohne Verhandlung, Mehrheitsentscheid oder hierarchische Steuerung - selbst über seine*ihre eigene Forschung trifft. Grundsätzlich gilt: Die Entscheidung, ein Thema wissenschaftlich zu bearbeiten, wird immer vor dem Hintergrund knapper Ressourcen und begrenzter Zeit gewählt. Jede Entscheidung zugunsten eines bestimmten Themas hat also zur Folge, dass weniger Zeit und Ressourcen für andere Themen zur Verfügung stehen.

Forschungsentscheidungen werden durch wissenschaftliche Akteur*innen selbst intentional im Rahmen einer Entscheidungssituation getroffen, die sich anhand der Akteur*innenkonstellation und der Interaktionsform charakterisieren lässt. Die Entscheidung, ein bestimmtes Thema aufzugreifen, weiterzuverfolgen oder aufzugeben, wird dabei selten aktiv, explizit, bewusst und strategisch getroffen - etwa: ,Ich habe freie Arbeitszeit. Beschäftige ich mich jetzt mit Klimawandel oder mit Schrumpfung?‘. Vielmehr manifestiert sich die Themenwahl von wissenschaftlichen Akteur*innen inkrementell in deren alltäglichen Einzelentscheidungen über ihre Forschungsprozesse und deren inhaltliche Ausgestaltung - etwa ,Nehme ich jetzt endlich die lange schon anvisierte Publikation zu Frage xy in Angriff?'; , Soll ich die Anfrage der angesehenen Fachkollegin, 
einen gemeinsamen Antrag zu stellen, annehmen, obwohl ich mittlerweile andere Fragestellungen spannender finde?'; ,Sollen wir das vorliegende Problem nach bewährtem Schema lösen oder wenden wir die neue Methode des Fachkollegen an? ‘; ,Lassen sich unsere Kompetenzen im Rahmen des neu aufgelegten Förderprogramms zu einem aussichtsreichen Antrag kombinieren?‘; ,Mit welchem Framing kann ich meinen Ergebnissen Aufmerksamkeit verschaffen?" oder "Soll ich die Anfrage, einen Vortrag über xy zu halten, annehmen und, wenn ja, wie gestalte ich diesen gewinnbringend?'.

Als Forschungsprozess ist dabei jedes zielgerichtete, einmalige und zeitlich begrenzte Vorhaben eines oder mehrerer wissenschaftlicher Akteur*innen zu verstehen, das unter Einsatz von Ressourcen durchgeführt wird, um wissenschaftliche Erkenntnisse zu einem bestimmten Thema zu produzieren und zu publizieren. Ein Forschungsprozess ist es daher auch, eine Publikation, einen Vortrag oder einen Forschungsantrag zu einer neuartigen Fragestellung zu entwickeln. Trotz der zur Bedingung erklärten Zielgerichtetheit, führt nicht jedes Projekt zu den anvisierten Erkenntnissen, nicht jede Forschung mündet in eine Publikation und nicht jede Erkenntnis entspricht dem ursprünglich fokussierten Zielhorizont.

In der wissenschaftlichen Einzelentscheidung evaluiert der*die Akteur*in selbst einerseits, welcher Beitrag zum Wissen der Gemeinschaft nachgefragt werden könnte, sowie andererseits, ob und in welcher Form er*sie diesen erzeugen kann und will. Dieser Mechanismus der autonomen Projekt- bzw. Themenwahl ist gemäß Gläser (2012b: 153-154) konstitutiv für eine effiziente Erweiterung des kollektiven Wissens:

\footnotetext{
„Die Produktion neuen Wissens ist ein kreativer Prozess, und die Formulierung einer für einen Wissenschaftler lösbaren Aufgabe setzt eine akkurate Einschätzung seiner kreativen Fähigkeiten voraus. Das kann aber nur der Betreffende selbst. Die ,SelbstIdentifikation'von Wissenschaftlern mit Aufgaben [...] erhöht die Wahrscheinlichkeit, dass diese an Aufgaben arbeiten, die sie auch tatsächlich lösen können. “
}

Akteur*innen treffen wissenschaftliche Einzelentscheidungen in wechselseitiger Anpassung zueinander, das heißt, sie handeln eigenständig in Kenntnis ihrer gegenseitigen Abhängigkeit und in rationaler Antizipation der Aktionen und Reaktionen der Fachgemeinschaft. Diese Interaktionsform entspricht einerseits der eines Netzwerks, denn ausschlaggebende Kriterien für die eigene Themenwahl werden - vermittelt über erwartete Belohnungen oder Sanktionen - aus der Wissensproduktion und den Wissenslücken der Fachgemeinschaft abgeleitet (bspw. ,Hat das schon mal jemand erforscht und wenn nein, kann ich damit einen 
interessanten Beitrag leisten?'). Allerdings beruht diese Netzwerkförmigkeit nicht allein auf persönlichen Beziehungen, sondern auch auf abstrakten Wissensbeziehungen, die sich aus der gegenseitigen Rezeption von Forschungsbeiträgen (ohne persönlichen Kontakt) ergeben. Die Interaktionsform hat andererseits marktförmigen Charakter, indem für die Produktion eigener Beiträge KostenNutzen-Erwägungen zum Tragen kommen und indem mitunter Angebot und Nachfrage in der kollektiven Wissensproduktion evaluiert werden, bevor ein eigenes Produkt eingebracht wird (bspw. ,Wäre ein eigener Forschungsantrag konkurrenzfähig?').

Die Themenwahl wird vor dem Hintergrund des institutionellen Kontextes getroffen, der primär die kollektive Wissensproduktion der entscheidungsrelevanten Fachgemeinschaft(en), aber auch systemexterne Einflussnahmen umfasst. Der institutionelle Rahmen schafft Bedingungen, Normen, Optionen, Anreize und Sanktionen für Forschungsentscheidungen. Im Rahmen der kollektiven Wissensproduktion der Fachgemeinschaft identifizieren die wissenschaftlichen Akteur*innen Lücken im gemeinsamen Wissen und formulieren Projekte zu deren Schließung. Alles, ,was andere diesem Wissensbestand schon hinzugefügt haben oder gerade hinzufügen möchten " (Gläser 2012b: 151), ist deshalb für die eigene Themenwahl und die mit der Themenwahl verbundenen Erwartungen von Bedeutung. Die kollektiv verhandelten Forschungsbedarfe werden über die Allokation von Reputation, materiellen Ressourcen und Positionen vermittelt. Wird ein Forschungsproblem seitens der Fachgemeinschaft als wichtig erachtet, so werden Ressourcen und Positionen dafür verfügbar gemacht und diejenigen Forscher*innen werden mit Reputation bedacht, die einen Beitrag zu dessen Lösung leisten. Diese auf der Meso- und Makroebene ansetzende Erklärung des Mechanismus fachgemeinschaftlicher Prioritätensetzung und -vermittlung lässt sich auch auf die Akteur*innenebene herunterbrechen und transepistemisch interpretieren: Die Sicherung der eigenen Position sowie des Sozialkapitals einer Akteurin bzw. eines Akteurs hängt auch davon ab, ob die verantworteten Allokationsentscheidungen im Sinne der (Präferenzen der) Fachgemeinschaft, der Fakultät oder dem editorial board einer Zeitschrift sind.

Die fachgemeinschaftliche Ressourcenallokation ist durch verschiedene Mechanismen vermittelt. So gerinnt die Präferenz der Fachgemeinschaft für ein bestimmtes Thema in einschlägigen Publikationen sowie in den Zitationen, die auf die einschlägigen Publikationen entfallen. Sie äußert sich in den tendenziell positiven Gutachten zu einschlägigen Publikationen und Forschungsanträgen sowie in Förderprogrammen mit dem thematischen Schwerpunkt vorausgesetzt die Gutachten und Konzeptionierungen liegen in der Hand der Fachgemeinschaft (wie bspw. bei Förderungen der DFG). An Hochschulen und 
Forschungseinrichtungen werden Strategien, Curricula sowie Denominationen leitender Positionen an aktuelle Relevanzkriterien angepasst. Einschlägige Arbeitskreise werden eingerichtet, Konferenzen und Sessions organisiert und besucht. Als Resultat eingeworbener Ressourcen leitender Wissenschaftler*innen werden Stellenausschreibungen veröffentlicht, die Nachwuchswissenschaftlerinnen und Nachwuchswissenschaftlern thematische Vorgaben machen. Diese Fokussierung von Ressourcen zugunsten eines bestimmten Themas (und zulasten anderer Themen) schlägt sich in der Aufmerksamkeitsverteilung der gesamten Fachgemeinschaft und den Entscheidungssituationen ihrer Mitglieder nieder. Weil die veränderten Belohnungsstrukturen für die Problemwahl für alle Mitglieder der Fachgemeinschaft mehr oder weniger sichtbar sind, bewerten viele wissenschaftliche Akteur*innen ihre Handlungsoptionen neu und gelangen gegebenenfalls zu neuen Entscheidungsergebnissen.

Zusätzlich zu diesem primären Arbeitskontext wissenschaftlicher Akteur*innen - der wissenschaftlichen Fachgemeinschaft - gibt es wissenschaftsexterne Faktoren, die zum einen die Rahmenbedingungen dieser kollektiven Wissensproduktion schaffen und modifizieren, die zum anderen aber auch direkt Einfluss auf die Entscheidungssituationen wissenschaftlicher Akteur*innen nehmen. Beispielsweise können aktuelle Problemlagen und Relevanzkriterien seitens der Gesellschaft an die Wissenschaft kommuniziert werden, sei es im direkten Austausch zwischen Wissenschaftler*innen und Akteur*innen der Gesellschaft oder vermittelt über die Massenmedien. Die direkte Einflussnahme vollzieht sich insbesondere durch die Bereitstellung materieller Ressourcen. Direkt können wissenschaftsexterne Akteur*innen Themen promovieren, indem sie bspw. im Rahmen von Ressortforschungseinrichtungen entsprechende politische Bedarfe durchsetzen, Forschungsfragen per Auftragsforschung vorgeben oder Förderprogramme auflegen, denen politische oder wirtschaftliche Relevanzkriterien zu Grunde liegen. Weitet ein*e Geber*in die Mittelzuweisung in einem Themenbereich aus, so steigen die für die einschlägige Forschung potenziell verfügbaren Ressourcen und mehr Forscher*innen beginnen, sich intensiv mit dem Thema zu beschäftigen. Dies wiederum lässt einschlägige Publikationen, Zitationen, Konferenzen etc. anschwellen, wodurch ein verdichteter Resonanzboden für thematisch passfähige Forschung entsteht. Die einschlägigen Forschungen bringen vermehrt Reputation ein, was wiederum die Chancen auf einflussreiche Positionen stärkt usw.

Die Einflussnahme wissenschaftsexterner Akteur*innen wirkt also nicht nur direkt auf die Entscheidungsoptionen einzelner Akteur*innen, wie etwa im Rahmen von Auftragsforschungen. Durch die direkte Beeinflussung der Themenwahl einzelner wissenschaftlicher Akteur*innen wird die kollektive Wissensproduktion 
und die fachgemeinschaftliche Sozialstruktur verändert, was indirekt wiederum Auswirkungen auf die durch die Fachgemeinschaft vermittelten Rahmenbedingungen und Relevanzkriterien hat. Die Wahl eines Themas durch eine*n wissenschaftliche*n Akteur*in hat also Auswirkungen nicht nur auf seine*ihre eigenen Präferenzen und Ressourcen (und damit auf seine*ihre zukünftigen Themenentscheidungen), sondern sie beeinflusst auch die kollektive Wissensproduktion der Fachgemeinschaft (und die durch sie vermittelten Themenpräferenzen) bzw. den institutionellen Kontext.

Indem das Erwerben von wissenschaftlichem Kapital die zentrale Handlungsorientierung wissenschaftlicher Akteur*innen darstellt, werden sie auch zu den maßgeblichen Selektionskriterien für die Wahl von Forschungsthemen. Ein*e wissenschaftliche*r Akteur*in präferiert also denjenigen Forschungsgegenstand, von dessen Bearbeitung er*sie sich den stärksten Kompetenzerwerb, den höchsten Reputationsgewinn, die meisten materiellen Ressourcen und den größten formalen Einfluss verspricht. Diese an die Themenwahl geknüpfte Erwartung entsteht auf Basis der persönlichen Interpretation der eigenen Fähigkeiten und Handlungsressourcen sowie des institutionellen Kontextes.

Die Handlungsorientierungen lassen sich nicht akteur*innenunabhängig gewichten und sind nicht gleichermaßen auf verschiedene Themenbereiche und Fachgemeinschaften beziehbar. Während einige Akteur*innen sich stärker durch Erfolge in der Drittmitteleinwerbung definieren, sehen andere Akteur*innen ihre Rolle eher als kritische Impulsgeber*innen. Handlungsleitende Interessen, normative Erwartungen und Identitätskonstruktionen leiten sich unter anderem aus der bisherigen Forschungsbiografie ab, das heißt aus den bislang verfolgten Schwerpunkten, Karrierestationen und den dementsprechend geprägten sozialen Beziehungen. Dies bringt eine inhaltliche Präferenz mit sich, denn fachfremde Kompetenz, Reputation, Drittmittel oder Positionen sind zwar nicht unbedingt schädlich, in der Regel aber auch kein persönliches Handlungsziel von Akteur*innen, zumal sie selten ohne einen unverhältnismäßig hohen Ressourceneinsatz zu erreichen sind. Die mit der Verfolgung eines Themas verbundenen Motivationen und Erwartungen unterscheiden sich also von Wissenschaftler*in zu Wissenschaftler*in und sind subjektiv.

Auch und insbesondere die Handlungsressourcen von Akteur*innen sind an bestimmte Themen gebunden und lassen sich nur in spezifischen Fachgemeinschaften realisieren. So lässt sich die Reputation, die ein*e Akteur*in aus seinen*ihren Veröffentlichungen schöpft, nur dort gewinnbringend einsetzen, wo eine thematische Verknüpfung mit den verfassten Inhalten für die Fachgemeinschaft erkenntlich ist. Genauso kann epistemisches Kapital, das durch die 
wissenschaftliche Tätigkeit in dem einen Bereich (bspw. Verkehrsplanung) akkumuliert wurde, in einem anderen Bereich (bspw. politische Ökonomie) wertlos sein, solange der*die Akteur*in keine inhaltliche Schnittmenge ausmachen bzw. konstruieren kann. Auch ökonomisches und politisches Kapital lässt sich nicht oder nur in begrenztem Maße in anderen Fachgebieten realisieren. Die eigene Forschungsbiografie bringt also eine einzigartige Kombination aus epistemischem, sozialem, politischem und ökonomischem Kapital in bestimmten Themenfeldern hervor, auf Basis derer sich eigene Präferenzen entwickeln, Handlungsoptionen entstehen und wahrgenommen werden und der institutionelle Rahmen interpretiert wird. Die thematisch gebundende Kombination wissenschaftlichen Kapitals wird in dieser Arbeit auch als Forschungsportfolio bezeichnet.

Wissenschaftliche Akteur*innen treffen die Wahl ihrer Forschungsthemen auf Basis der subjektiven Interpretation nicht nur ihrer individuellen Voraussetzungen und der Entscheidungssituation, sondern auch der kollektiven Wissensproduktion. Hierzu gehören zum einen die Identifizierung von Forschungsbedarfen und Handlungsoptionen sowie zum anderen die Bewertung der eigenen Ressourcen vor dem Hintergrund der jeweiligen Fachgemeinschaft. Ein wichtiger Faktor dabei ist, ob sich aus den eigenen Handlungsressourcen Wettbewerbsvorteile in der Fachgemeinschaft ergeben. Aussagen aus zwei von Latour und Woolgar (1986: 190) durchgeführten Interviews veranschaulichen diese Sichtweise:

„This instrument can bring me ten papers a year"

„Why working on this [substance], we are not the best in this area; we invested a lot in the releasing factor field ... we are the best in it, we'd better stay in it"

In kleinen Fachgemeinschaften, die sich um ein neues Forschungsproblem gruppieren, sind solche Vorteile leichter zu realisieren, als in etablierten Fachgemeinschaften, die bereits auf einen ausdifferenzierten Wissenskatalog verweisen können. Während erstere offener für neue Mitstreiter*innen und Impulse sind, setzen letztere eine hohe Eintrittshürde, indem die Kenntnis des fachgemeinschaftlichen Wissens vorausgesetzt wird. Das Aufgreifen eines bislang wenig erforschten Themas ist dementsprechend verbunden mit der besseren Chance, einen (in der Nische) relevanten Beitrag zu leisten. Ein womöglich gleichbleibend kleines Publikum setzt aber andererseits auch eine natürliche Grenze für den möglichen Reputationserwerb. Demzufolge kann das Aufgreifen eines bislang wenig erforschten, aber voraussichtlich an fachöffentlicher Aufmerksamkeit gewinnenden Themas unter Kosten-Nutzen Erwägungen insbesondere für junge Wissenschaftler*innen attraktiv erscheinen. 
Aufgrund der thematisch gebundenen, akkumulierten Handlungsressourcen und der herausgebildeten Handlungsorientierungen sind Themenentscheidungen also in hohem Maße pfadabhängig. Sich ein gänzlich neues Thema zu erarbeiten und dort einen relevanten Fachbeitrag einbringen zu können, ist für eine*n Wissenschaftler*in mit großem Aufwand verbunden. Umso mehr (themengebundenes) Kapital ein*e Akteur*in bereits in anderen Themenbereichen akkumuliert hat, umso mehr wird er*sie diesen Aufwand scheuen. Identifiziert ein*e etablierte*r Akteur*in Parallelen oder Schnittmengen zwischen einem von ihm*ihr bereits erforschten Thema und einem neuen Thema, so ist der Aufwand geringer und Wettbewerbsvorteile in dem neuen Fachbereich sind für ihn eventuell bereits erkennbar. Ein Fachbeitrag oder Forschungsantrag, in dem er bspw. sein bereits vorhandenes Wissen mit einem neuen Thema verknüpfen kann, könnte dann unter Kosten-Nutzen-Erwägungen Sinn ergeben, weil der*die Akteur*in mit relativ geringem Aufwand eine neue Quelle für soziales, ökonomisches und politisches Kapital erschließen kann. Forscher*innen, die in der bisherigen Forschungsbiografie nur wenige Handlungsressourcen akkumuliert haben, die also in allen Themen ein ähnliches Maß an Handlungsressourcen zur Verfügung haben, sind in ihrer Themenwahl weniger durch ihre bisherige Karriere geleitet bzw. weniger pfadabhängig. Eine Ausnahme stellen Akteur*innen dar, die eine gewisse Sättigung feststellen, die also das Gefühl haben, genug Wissen zu einem bestimmten Thema produziert zu haben oder ihr Maximum an Reputation in dem Bereich erreicht zu haben.

Zur Veranschaulichung wird in Abb. 5.3 die wissenschaftliche Themenwahl in vereinfachter Form bildhaft dargestellt. Sie orientiert sich an dem analytischen Modell des Akteurzentrierten Institutionalismus (siehe Abb. 5.1), fokussiert aber auf die Einzelentscheidung der Themenwahl wissenschaftlicher Akteur*innen, was einige Detail-Betrachtungen wichtiger Mechanismen ermöglicht. Die wichtigste Modifikation gegenüber Mayntz und Scharpf besteht in der Handhabung der Systemumwelt und des institutionellen Kontextes. Sie ergibt sich aus der Selbstreferenzialität der Wissenschaft: Die Wissenschaft ist ihr eigenes Publikum und trifft Entscheidungen (bspw. im Gegensatz zur Politik) nicht für eine systemexterne Umwelt (bspw. das Wirtschaftssystem).

Jede wissenschaftliche Entscheidung (15) betrifft dementsprechend anstelle der „Umwelt“ zunächst das eigene Forschungsprogramm (13), was sich wiederum auf die eigene Kapitalstruktur und Handlungsorientierungen auswirkt (16, 11). Zudem wirkt sich jede wissenschaftliche Kommunikation (14) des Akteurs bzw. der Akteurin (17) auf die fachgemeinschaftliche Wissensproduktion (4) aus, indem er*sie bspw. eine Forschungslücke schließt oder durch Zitieren Reputation verteilt (10). Die kollektive Wissensproduktion ist gleichzeitig ein Teil des 


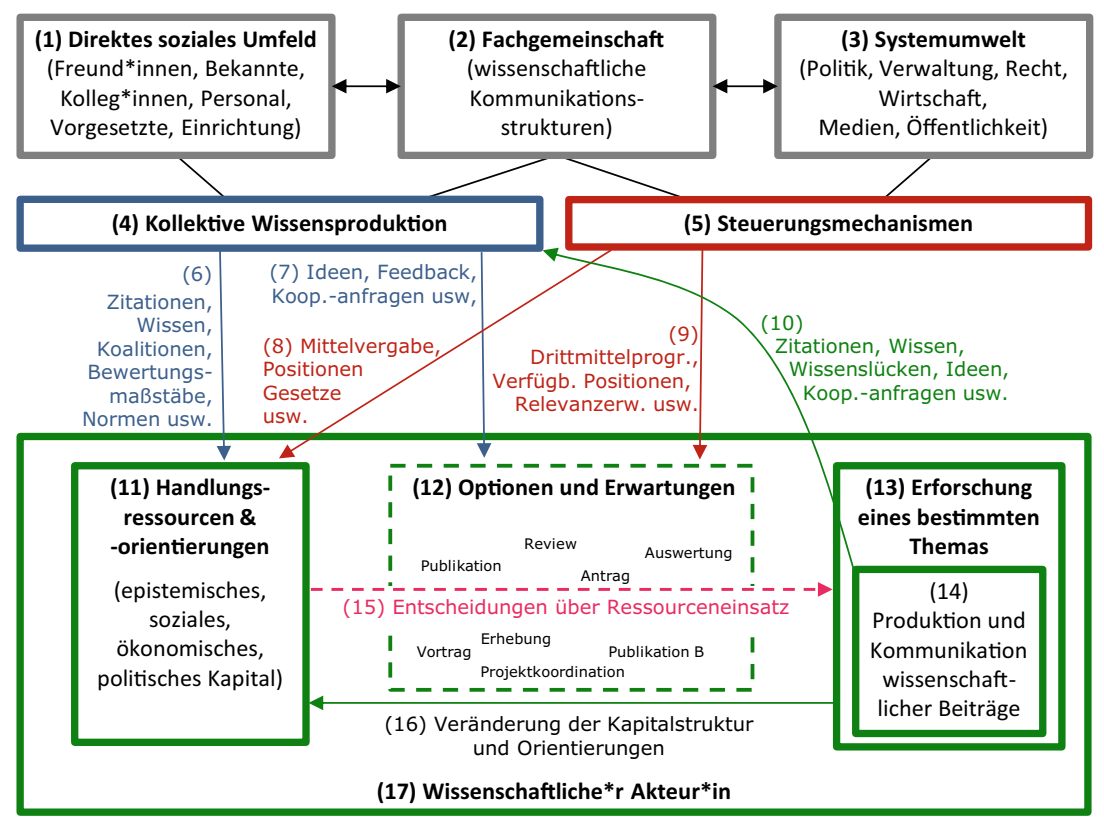

Abb.5.3 Themenwahl wissenschaftlicher Akteur*innen (Eigene Darstellung)

institutionellen Kontextes, indem durch sie Reputation verteilt oder Relevanzkriterien vermittelt werden (6) und indem sie Handlungsoptionen hervorbringt $(7,12)$. Der institutionelle Kontext besteht darüber hinaus aus den formellen Steuerungsmechanismen (5), die über Gesetzgebung, Drittmittelvergabe oder die Besetzung von Positionen ebenfalls Handlungsressourcen und -optionen vergeben $(8,9)$. Die Steuerungsmechanismen werden sowohl durch die Fachgemeinschaft (2) als auch durch die Systemumwelt (3) bestimmt. Die kollektive Wissensproduktion wird dagegen nur indirekt durch die Systemumwelt beeinflusst. Allerdings wird auf der Seite der kollektiven Wissensproduktion eine weitere Differenzierung vorgenommen: Das direkte soziale Umfeld (1), das zumindest teilweise eine Teilgruppe der Fachgemeinschaft darstellt, wird gesondert aufgeführt, um dessen Bedeutung herauszustellen. Schließlich sind wissenschaftliche Einzelentscheidungen in besonderem Maße von den Bewertungsmaßstäben befreundeter Kolleg*innen abhängig. Auch sind für die Entstehung neuer Ideen direkte Austausche - das zeigt die Empirie dieser Arbeit - häufig wichtiger als 
die abstrakte Wissensproduktion auf der Grundlage der Produktion und Rezeption von Publikationen.

Mit diesen Kategorien und Mechanismen der Themenwahl ist der analytische Rahmen zur Analyse von Themenkarrieren in der Wissenschaft vollständig. Wie die erläuterten Aspekte im Rahmen der Empirie erhoben werden, zeigt das folgende Kapitel.

Open Access Dieses Kapitel wird unter der Creative Commons Namensnennung 4.0 International Lizenz ( http://creativecommons.org/licenses/by/4.0/deed.de) veröffentlicht, welche die Nutzung, Vervielfältigung, Bearbeitung, Verbreitung und Wiedergabe in jeglichem Medium und Format erlaubt, sofern Sie den/die ursprünglichen Autor(en) und die Quelle ordnungsgemäß nennen, einen Link zur Creative Commons Lizenz beifügen und angeben, ob Änderungen vorgenommen wurden.

Die in diesem Kapitel enthaltenen Bilder und sonstiges Drittmaterial unterliegen ebenfalls der genannten Creative Commons Lizenz, sofern sich aus der Abbildungslegende nichts anderes ergibt. Sofern das betreffende Material nicht unter der genannten Creative Commons Lizenz steht und die betreffende Handlung nicht nach gesetzlichen Vorschriften erlaubt ist, ist für die oben aufgeführten Weiterverwendungen des Materials die Einwilligung des jeweiligen Rechteinhabers einzuholen. 